\title{
Zur Frage informeller Machtverhältnisse im Staatssozialismus. Das Beispiel der tschechoslowakischen Industriebetriebe 1945-1968
}

\begin{abstract}
Peter Heumos*
Es gehört zu den gesicherten Ergebnissen der sozialgeschichtlichen Kommunismusforschung, dass die kommunistischen Parteien in den Ländern des sowjetischen Imperiums keine wirklich effektive Kontrolle über den Produktionsprozess ausübten. Andererseits wurde der dadurch entstehende organisatorische, soziale und politische Freiraum von den Industriearbeitern unterschiedlich genutzt. In der Tschechoslowakei setzte dieser Freiraum zwischen 1945 und 1968 einen Prozess der Selbstorganisation und des innerbetrieblichen Machterwerbs der Industriearbeiter in Gang, der beträchtliche Dimensionen erreichte und daher auch den Realitätskontakt der gängigen totalitarismustheoretischen Interpretationen in Zweifel zieht.

1. Im April 1953 stand es schlecht um die monatliche Planerfüllung in den Vereinigten Stahlwerken Kladno. Der Direktor der Werke nannte einem Emissär des Ministeriums für Hüttenindustrie und Erzgruben die Gründe: Vor allem gehe es mit dem sozialistischen Wettbewerb nicht voran, dem Motor der Planerfüllung. Die Materialzufuhr sei unregelmäßig, die Auftragslage decke nicht alle Werksabteilungen ab, die künftige Auslastung der einzelnen Produktionsbereiche sei wegen einer schwebenden Entscheidung der Planungsbehörde über eine Senkung des Plansolls offen. Der Emissär beruhigte den besorgten Direktor. Die Stahlwerke sollten nicht versuchen, bei der Planerfüllung „Wunder zu vollbringen“, denn Luppen ${ }^{1}$ und Schrott für die Hochöfen seien rar. Einige Tage vor diesem Gespräch hatten Partei und Regierung einen „mobilisierenden“ Beschluss zum Plansoll der Stahlwerke Kladno gefasst. ${ }^{2}$

Der Emissär in Kladno war keine Einzelerscheinung, er stand für ein System informeller Kontakte der Mitarbeiter der Ministerien und Zentralbehörden, die „die Betriebe bereisen und sich bemühen, einen Überblick darüber $\mathrm{zu}$ gewinnen, was in den Betriebsversammlungen kritisiert wurde". ${ }^{3}$

Auch in marktförmigen Gesellschaften sind formelle bzw. vertragliche Beziehungen offenbar nur dann funktionsfähig, wenn sie durch informelle Beziehungen gestützt werden, wie neuere Diskussionen über „Einbettung“ und „Moralökonomie“ zeigen. ${ }^{4}$ Netzwerke im Staatssozialismus erreichten ein solches Ausmaß, dass sie als „Vergesellschaftungsform“ bezeichnet werden können. ${ }^{5}$ Es gibt bekanntlich gute Gründe, ihre funktionale Notwendig-

\footnotetext{
* Dr. Peter Heumos - bývalý odborný pracovník; Collegium Carolinum, München (janaheumos@yahoo.de).

1 Lockere Eisenklumpen, ein Zwischenprodukt bei der Verarbeitung von Roheisen zu schmiedbarem Eisen.

2 Všeodborový archiv ČMKOS (künftig zitiert: VOA), Prag. ÚRO-VMP, Karton 2, 1953, Berichte der Brigaden und Berichte über sozialistische Wettbewerbe, SONP Kladno.

3 Národní archiv (künftig zitiert: NA), Prag. Fonds 014/12, Bd. 23, Nr. 822, 1956/5. Sozialgeschichte der DDR. Hrsg. von Kaelble, H. - Kocka, J. - Zwahr, H., Stuttgart 1994, 31-61.

5 Ebd., 37.
}

4 Kohli, M. (1994): Die DDR als Arbeitsgesellschaft? Arbeit, Lebenslauf und soziale Differenzierung, in:
\end{abstract}


keit gerade im Hinblick auf die Problematik des Plans hervorzuheben, denn gegen ein starres, hierarchisch organisiertes Anweisungs- und Zuteilungsverfahren gewährleisteten die informellen sozialen Interaktionen eine gewisse innere Elastizität des Plans. Jahrelang regnete es in die Gießerei der Pilsener Škoda-Werke, aber die Betriebsleitung konnte die Mittel für die Reparatur des Daches nicht auftreiben. ${ }^{6}$ In Borovany dagegen führte der Direktor einer Lebensmittelfabrik einen erfolgreichen Kampf gegen das chronische Defizit der staatlichen Zuwendungen für Instandsetzungsarbeiten: Geld für derartige Zwecke floss, solange er sich der Gunst seiner „Bekanntschaften im Ministerium“ erfreute.

Für Informalität dieser Art wird auch der Begriff „Planerfüllungspakt“" benutzt. ${ }^{8}$ Dieser bezeichnet das Vorverständigtsein der beteiligten Akteure darüber, dass der politische Imperativ der Planerfüllung eine Sache war, die realen Verhältnisse eine andere. Letztere aber zwangen zur informellen Praxis eines Stillhalteabkommens zumeist auf der Grundlage von Tauschbeziehungen.

Unter tschechoslowakischen Bedingungen stand auf der einen Seite außer Frage, dass die von den Planungsbehörden veranlassten häufigen Wechsel der Produktionsprogramme die Betriebe immer wieder in allergrößte organisatorisch-technische Schwierigkeiten stürzten, ${ }^{9}$ dass die ständige Ausweitung der Produktion bei konstanter Produktionsfläche zu einer fortschreitenden Verringerung der gesetzlich vorgeschriebenen Größe des Arbeitsplatzes und zu unzumutbaren Belastungen und hoher Unfallhäufigkeit führte, ${ }^{10}$ dass die Arbeiter durch die Unstetigkeit der Produktion aufgrund schwankender Materiallieferungen auch materiell starke Einbußen erleiden konnten, ${ }^{11}$ dass die allgemeinen Arbeitsbedingungen angesichts häufig fehlender Sicherheits-, sozialer und hygienischer Einrichtungen und verbreiteter technischer Rückständigkeit im Ganzen miserabel waren. ${ }^{12}$

Auf der anderen Seite hielt die Wirtschaftsbürokratie in Prag für diese Defizite einige Entschädigungen bereit; dazu gehörte in erster Linie ihr Umgang mit dem zentralen Problem der Löhne und Normen. Bis in die späten fünfziger Jahre übten die Betriebe erheblichen Einfluss auf den Lohnfestsetzungsprozess aus, da der Anteil des Tariflohns am Lohn nach 1948 auf 40-50 Prozent absank ${ }^{13}$ und die beweglichen Teile des Lohns (Prämien, Zuschläge, Überstunden etc.) im Wesentlichen auf innerbetrieblichen Aushandlungsprozessen beruhten - unter wohlwollender Duldung der Wirtschafts-

6 Škoda-Archiv, Pilsen. ROH 15/PV 163, Protokoll der Plenarsitzung des Betriebsausschusses Hütte und der Vorsitzenden der Werkstatt-Ausschüsse am 8. 2. 1956.

7 VOA. KOR, Karton 75/1956, Inventarnummer 138/1, Bewertung der allgewerkschaftlichen Aktive der Bezirksfunktionäre vom 6. bis 20. 7. 1956 in allen Bezirken des Kreises České Budějovice.

8 Entwickelt wurde der Begriff am Beispiel der DDR. Vgl. Voskamp, U. - Wittke, V. (1990): Aus Modernisierungsblockaden werden Abwärtsspiralen. Zur Reorganisation von Betrieben und Kombinaten in der ehemaligen DDR, in: SOFI-Mitteilungen Nr. 18 (1990) 12-30.

9 Die Belege dafür sind massenhaft, nur ein Beispiel sei herausgegriffen: VOA. ÚRO-Org., Karton 103, Inventarnummer 353, Auszug aus den Meldungen der Instrukteure der Kreisgewerkschaftsräte für den Genossen L. Novák vom 23. 2. 1950.

10 VOA. ÚVOS-Spotřebný průmysl, Faszikel 4, 1965, Erfahrungen des ZK des Gewerkschaftsverbandes der Beschäftigten der Konsumgüterindustrie mit der Tätigkeit der Gewerkschaftsorganisation des Nationalunternehmens Svit Gottwaldov.

11 Die Kollektivprämie für die Planerfüllung bei den Bergleuten im slowakischen Rožňava lag 1953 bei 800 bis 1000 Kronen. Der durchschnittliche monatliche Nettoverdienst in den oberen Tarifklassen betrug 2000 bis 3500 Kronen. VOA. Celostátní akce, Karton 3, Inventarnummer 8 b, Beratung der Vorsitzenden und Organisationssekretäre der Kreisgewerkschaftsräte am 12. 10. 1953.

12 Für 1956 vgl. dazu den gemeinsamen Bericht der Abteilung „Arbeitsschutz und Arbeitssicherheit“ und der Abteilung „Nationalversicherung“ des ZK des Gewerkschaftsverbandes der Beschäftigten im Maschinenbau über risikoreiche und technisch schlecht ausgestattete Arbeitsplätze in Maschinenbaufabriken. VOA. Strojírenství, Karton 27/1956.

13 Škoda-Archiv. PV KSČ 3/235, Protokoll der Sitzung des technisch-ökonomischen Rates des Unternehmens am 17. 12. 1958 
verwaltung. Prämienordnungen beispielsweise gaben sich die Betriebe häufig selbst, ohne offizielle Zustimmung oder korrigierendes Eingreifen der Ministerien bzw. der so genannten Hauptverwaltungen, die dann auch nur selten die Wirksamkeit und ökonomische Zweckmäßigkeit der Prämienordnungen kontrollierten. Die Folge war, dass um die Mitte der fünfziger Jahre im Maschinenbau rund 3 000, in der chemischen Industrie 2000 und in der Bauindustrie ca. 600 Prämienordnungen existierten ${ }^{14}$ - die Hauptursache der bunten Vielfalt der Lohnverhältnisse im tschechoslowakischen Staatssozialismus gerade der stalinistischen Periode. ${ }^{15}$ "Die gleiche Arbeit wird überall anders bezahlt," hieß es noch 1956 unter den Arbeitern. ${ }^{16}$ Ebenso indifferent verfuhren die zentralen Behörden in der Frage der Arbeitsnormen, die sich als ungelöstes Problem durch unseren Untersuchungszeitraum hinzieht. ${ }^{17}$ Die Gewerkschaftsführung hielt sich mit Kritik nicht zurück, bezweifelte den ernsthaften Willen der Bürokraten, mit der massenhaften „Unsitte“ fiktiver (,weicher") Normen aufzuräumen, an denen alle Reformen des Lohnsystems scheiterten. ${ }^{18}$

Man wird einwenden, hier gehe es nicht um Indifferenz der Bürokratie, sondern darum, dass sich diese mit der "Staatsklasse“ aus politisch-ideologischen Gründen nicht anlegen konnte. Plausibel ist das nur dann, wenn man die Bürokratie im Staatssozialismus für ein „bloßes Instrument einer im Prinzip nicht kalkulierbaren Macht" hält. ${ }^{19}$ So einfach war die Sache nicht. Wurden Grundüberzeugungen ihres traditionellen Selbstverständnisses berührt, legte sich die tschechoslowakische Wirtschaftsbürokratie quer zur Programmlinie der KPTsch. Das galt etwa für die politisch besonders hoch gehängten Formen der Amalgamierung von Ökonomie und Politik, also vor allem für den sozialistischen Wettbewerb. Auf der Grundlage informeller Verständigung mit den Betrieben verfuhr die Ministerialbürokratie auf diesem Gebiet in einer Weise, die sich nur als Boykott bezeichnen lässt. ${ }^{20}$ Auch der Fusionierung von Technikentwicklung und politischer Mobilisierung im Rahmen der Novatorbewegung setzten die Behörden eine massive Verschleppungstaktik entgegen, hinter der die von vielen Betriebsräten mitgetragene Forderung stand, die Betriebstechniker aus dieser Bewegung auszuschließen, da technische Verbesserungen zu deren dienstlichen Pflichten gehörten. ${ }^{21}$ Erfindungen im Rahmen der Novatorbewegung mochten Aufsehen erregen und von Partei und Gewerkschaften auf spektakuläre Weise gefeiert werden - die Experten in den zentralen Behörden blieben ungerührt: Sie würden keine Erfindung akzeptieren (und honorieren), über die man sich schon „bei Leonardo da Vinci“ informieren könne. ${ }^{22}$

14 VOA. ÚRO-Sekr., Karton 76, Inventarnummer 672, 1955, Bericht über die Maßnahmen zur Beseitigung von Mängeln bei der Entlohnung der Arbeit und zum Entwurf der politisch-organisatorischen Absicherung dieser Maßnahmen.

15 Zahlreiche Beispiele dazu bei Heumos, P. (2001): Aspekte des sozialen Milieus der Industriearbeiterschaft in der Tschechoslowakei vom Ende des Zweiten Weltkrieges bis zur Reformbewegung der sechziger Jahre, in: Bohemia 42 (2001) 323-362.

16 VOA. KOR, Karton 75/1956, Inventarnummer 138/1, Bewertung der allgewerkschaftlichen Aktive der Bezirksfunktionäre vom 6. bis 20. 7. 1956 in allen Bezirken des Kreises České Budějovice.

17 VOA. Strojírenství, Karton 10, Faszikel 1, 1954, Wie das Kreiskomitee des Gewerkschaftsverbandes für schweren Maschinenbau in Liberec die gleichmäßige Erfüllung des Planes in seinen Betrieben sicherstellt und wie es die Steigerung der Arbeitsproduktivität im Hinblick auf das Wachstum der Löhne und Gehälter verfolgt (14. 3. 1953).

18 VOA. Celostátní akce, Karton 5, Inventarnummer 32, Stenographisches Protokoll der Beratung der Vorsitzenden der ROH-Verbände und der Abteilungsleiter des Zentralrates der Gewerkschaften am 17. 1. 1955.

19 Zit. nach Srubar, I. (1991): War der reale Sozialismus modern? Versuch einer strukturellen Bestimmung, in: Kölner Zeitschrift für Soziologie und Sozialpsychologie 43 (1991) 415-432.

20 VOA. ÚRO-Sekr., Karton 46, Inventarnummer 599, Bericht über die Entwicklung des sozialistischen Wettbewerbs und über den Vorschlag zur Verleihung der Roten Fahnen im vierten Quartal 1953 für das Sekretariat des Zentralrates der Gewerkschaften vom 27. 1. 1954.

21 VOA. OS Kovo, Karton 4 A, Faszikel 4, 1951, Stand des sozialistischen Wettbewerbs und der Novatorbewegung im Betrieb ČKD Sokolovo.

22 Das Zitat aus der Stellungnahme des Ministeriums für Bauwesen zu einer Erfindung, die 1955 zum 38. Jahrestag der russischen Oktoberrevolution mit einem Stafettenlauf von Trutnov nach Prag gefeiert wurde. VOA. 
Die Netzwerke zwischen der Wirtschaftsbürokratie und den Betrieben glichen also nicht nur Mängel des Systems aus, sondern brachten auch Interessen mit geringer Konformität zu diesem ins Spiel brachten. Das tritt noch deutlicher hervor, wenn man das informelle Geflecht des Planerfüllungspaktes auf der Ebene des Betriebes betrachtet. In dieser Perspektive sollte jedenfalls für die Tschechoslowakei die an den gesellschaftlichen Verhältnissen der DDR gewonnene Vorstellung revidiert werden, die informellen innerbetrieblichen Regelungen seien lediglich eine ,passive Stärke“ der Belegschaften gewesen. ${ }^{23}$

2. Bereits vor dem Februar 1948) waren die bürokratisch-administrativen Regelungen der Organisation des Produktionsprozesses weit davon entfernt, auf die shop-floor-Ebene durchschlagen zu können. Die Kontrolle über die Normierung des Arbeitsprozesses, über Leistung und Lohn lag im Wesentlichen bei den Arbeitern. Das war eine Folge der Machtstellung der Betriebsräte, die nach dem Krieg mit einem libertären, antistaatlich eingefärbten Sozialismus-Konzept aus dem Untergrund auftauchten und ohne deren Zustimmung sich in den frühen Nachkriegsjahren in den Betrieben „keine Maus“ regte. ${ }^{24}$ Obwohl die Position der Räte durch die 1945 gegründete Einheitsgewerkschaft eingeengt wurde, blieben diese stark genug, um das betriebliche Milieu durch zwei Zielvorstellungen auf Dauer zu prägen: durch die in ihrer Intensität zwar schwankende, aber nie von der Tagesordnung genommene Forderung nach Dezentralisierung der verstaatlichten Industrie und durch eine rigoros praktizierte egalitäre Lohn- und allgemeine Sozialpolitik.

Für den Rückbau des industriellen Zentralismus wurden - wie überall im Realsozialismus - tagtäglich Argumente geliefert. Am grünen Tisch könne man leicht entscheiden, aber im Betrieb sehe die Sache ganz anders aus, schrieb der gewerkschaftliche Betriebsausschuss der Pilsener Škoda-Werke an den Zentralrat der Gewerkschaften. ${ }^{25}$ Die Parteiorganisation im Betrieb müsse das ,ausbügeln“, was „die oben vermasselt haben,“ sagte der Vorsitzende des Betriebskomitees der KPTsch im Eisenbahndepot in Veselí n.L. ${ }^{26}$ Daraus folgte eine innerbetriebliche informelle Praxis, die sich wenig um Gesetze und Verordnungen scherte. Das Maß aller Dinge war die betriebliche Realität. „Eure Richtlinien taugen nichts,“ hielt ein Betriebsratvorsitzender in Ružomberok einem höheren Gewerkschaftsfunktionär vor, „wir müssen das hier im Betrieb so machen, dass es unseren Bedingungen entspricht. “227

Regulative Idee des innerbetrieblichen Beziehungsnetzwerks war der Egalitarismus der Betriebsräte vor allem in der Lohnpolitik, den die Gewerkschaften seit 1948 als „Gleichmacherei“ skandalisierten ${ }^{28}$ und den die KPTsch aus politischen Gründen - um ihren Rückhalt in den unteren Einkommensschichten der Arbeiterschaft nicht zu verlieren - bis zur Mitte der sechziger Jahre mit zusammengebissenen Zähnen tolerierte. ${ }^{29}$

Strojírenství, Karton 35-36, Faszikel 4, 1957, Stenographisches Protokoll der gesamtstaatlichen Beratung der Erfinder, Verbesserer und Novatoren auf der Prager Burg am 24. 10. 1957.

23 Zit. nach Kohli, Die DDR als Arbeitsgesellschaft, 49.

24 Heumos, P. (1981): Betriebsräte, Einheitsgewerkschaft und staatliche Unternehmensverwaltung. Anmerkungen zu einer Petition mährischer Arbeiter an die tschechoslowakische Regierung vom 8. Juni 1947, in: Jahrbücher für Geschichte Osteuropas 29 (1981) 215-245.

25 Škoda-Archiv. ROH 8/PV 544, Schreiben des Betriebsausschusses der ROH der Lenin-Werke [= Škoda-Werke] an den Zentralrat der Gewerkschaften vom 15. 1. 1958.

26 NA. Fonds 014/12, Bd. 15, Nr. 377, Bericht über die Einführung des neuen Lohnsystems auf den tschechoslowakischen Staatsbahnen (Oktober 1954).

27 VOA. ÚRO-Org., Karton 105, Inventarnummer 382, Bericht des Instrukteurs des Kreisgewerkschaftsrates Žilina Karol Pistovčák [November 1951].

28 Vgl. dazu den Bericht der Abteilung „Arbeit und Löhne“ des Kreisgewerkschaftsrates Liberec für den Zentralrat der Gewerkschaften vom 26. 1. 1951. VOA. Faszikel „Normenüberprüfung auf der Grundlage des Regierungsbeschlusses vom 14. 7. 1950“ (nicht inventarisiertes Material).

29 Kaplan, K. (1993): Sociální souvislosti krizí komunistického režimu v letech 1953-1957 a 1968-1975. Praha 1993, 22 und 27 (Sešity Ústavu pro soudobé dějiny 9). 
Um diesen Egalitarismus zu verwirklichen, bedurfte es entsprechender Machtverhältnisse im Betrieb, die unten noch dargestellt werden sollen. Hier sei zunächst auf die wichtigsten lohnpolitischen Strategien der gewerkschaftlichen Betriebsorganisationen hingewiesen. Den Kern bildete die Forderung nach „Beseitigung des sozialen Unrechts, das bisher vor allem an den sozial schwächsten Arbeitern begangen wurde,“ wie es die Gewerkschafter in den Brünner Waffenwerke im Juli 1945 formulierten. ${ }^{30}$ Das Rezept waren „weiche“ Normen für die Arbeiter der unteren Tarifklassen, die leicht überschritten werden konnten und damit besseren Verdienst ermöglichten. ${ }^{31}$ Ebenso wurden Prämienordnungen dazu verwendet, die Verdienstunterschiede zwischen Hilfs- und Facharbeitern auszugleichen ${ }^{32}$ und die Arbeitseinkommen von Arbeitern und technisch-administrativem Personal zu nivellieren. ${ }^{33}$ Unzählige Male protestierten die gewerkschaftlichen Organisationen in den Betrieben zwischen 1945 und 1968 gegen die nach ihrer Auffassung $\mathrm{zu}$ hohen Gehälter des industriellen Managements: Ein „Zweiter Kapitalist“ als Betriebsdirektor sei unerwünscht. ${ }^{34}$ Besonders der sozialistische Wettbewerb bot Möglichkeiten zuhauf, um den kollektiven Gleichheitsanspruch zu praktizieren. Individuelle Leistung wurde dort in großem Maßstab zugunsten eines kollektiven Verteilungsmusters unterlaufen. Wie in zahllosen anderen Betrieben organisierten die Arbeiter im Škoda-Zweigwerk Smíchov Wettbewerbe um den „,besten Arbeiter des Betriebes“ so, dass jeder in einem festen Turnus einmal an die Reihe kam und die Prämie erhielt. ${ }^{35}$ Auch Wettbewerbe zwischen einzelnen Werkstätten eines Betriebes folgten diesem Grundsatz. ${ }^{36}$ Mährische Sägewerk-Arbeiter fanden schließlich im Frühjahr 1956, sie seien alle „die besten Arbeiter“ und die Auszeichnung zum besten Arbeiter sei „Personenkult“: Also wurden Wettbewerbe und Auszeichnungen im Betriebsrat abgewählt. ${ }^{37}$

Dass sich dieses Geflecht informeller Regelungen auf Dauer etablieren konnte, setzte „entgegenkommende“ betriebliche Machtverhältnisse voraus. Damit ist die Position des industriellen Managements ${ }^{38}$ angesprochen.

In der Erwartung, unter kommunistischer Herrschaft werde es autoritär zugehen, pflegte das industrielle Management nach 1948 zunächst die Betriebsräte als Sprachrohr „,unverantwortlicher Forderungen“ bei den Ministerien anzuschwärzen, ${ }^{39}$ und versuchte obendrein, wie in den Stahlwerken Kladno, sich der Parteiführung durch das Angebot

30 VOA. ÚRO-Soc., Karton 1, Inventarnummer 1/6, 1945, Gemeinsame Resolution des Betriebsrates und der betrieblichen Gewerkschaftsgruppe der Brünner Waffenwerke vom 12. 7. 1945.

31 VOA. Strojírenství, Karton 65, 1963, Protokoll der Sitzung des Betriebsausschusses der ROH [der Tschechoslowakischen Dieselmotorenwerke in Smíchov] am 13. 5. 1963.

32 VOA. ÚRO-Před., Karton 56, Inventarnummer 355 III/3, Materialien für die Sitzung des Präsidiums des Zentralrates der Gewerkschaften am 31. 10. 1961: Bericht über die Erfahrungen bei der Anwendung von Kollektivprämien vom 19. 9. 1961

33 Vgl. dazu den Auszug aus dem Bericht des Kreiskomitees der KPTsch in Pilsen über die Prämienpolitik in den Škoda-Werken (Juli 1953). NA. Fonds 014/12, Bd. 11, Nr. 186.

34 Škoda-Archiv. ROH 4/PV 696, Protokoll der Plenarsitzung des Unternehmensausschusses und der Vorsitzenden der Betriebsausschüsse der ROH am 1. 7. 1958.

35 VOA. ÚRO-VMP, Karton 2,1953, Berichte der Brigaden und Berichte über sozialistische Wettbewerbe, Betrieb Škoda-Smíchov.

36 Škoda-Archiv. ZVIL 101/400 a, Wettbewerb auf Unternehmensebene - die beste Betriebsabteilung im Dezember 1955.

37 VOA. KOR, Karton 78/1956, Inventarnummer 141, Bericht über die Verwirklichung der Thesen des ZK der KPTsch zur weiteren technischen Entwicklung in den Betrieben des Kreiskomitees des Gewerkschaftsverbandes der Holzindustrie vom 14. 6. 1956.

38 Mit der Geschichte des industriellen Managements hat sich systematisch bisher nur Lenka Kalinová befasst. Von ihren zahlreichen Studien zu dieser Thematik sei an dieser Stelle nur die jüngste genannt: Ke změnám ve složení hospodářského aparátu ČSR v 50. letech, in: Stránkami soudobých dějin. Sborník statí ke pětašedesátinám historika Karla Kaplana, ed. Jech, K.. Praha 1993, 149-157.

39 VOA. NHK, Karton 82, Inventarnummer 219, Bericht [des Nationalunternehmens] der Tschechoslowakischen Hüttenindustrie (August 1948). 
anzudienen, selbst in den Betrieben mit repressiven Mitteln für Ruhe und Ordnung sorgen $\mathrm{zu}$ wollen. ${ }^{40}$ In der Folgezeit musste das betriebliche Leitungspersonal allerdings Erfahrungen machen, die schließlich zu einem widerspenstigen Sich-Arrangieren mit den herrschenden Verhältnissen führten. Bei Streiks wurde die Betriebsleitung mit einer gewissen Regelmäßigkeit zur Rechenschaft gezogen, ${ }^{41}$ und obwohl bereits 1950 ein Drittel aller Betriebsdirektoren aus der Arbeiterschaft $\mathrm{kam}^{42}$ konnte sich das industrielle Management nicht sicher sein, bei der nächsten politischen Säuberung ungeschoren davonzukommen. ${ }^{43}$ Die hohe Fluktuation der betrieblichen Leitungskader gehörte denn auch zu den beliebten Themen des Volkswitzes. ${ }^{44}$

Die Literatur präsentiert den tschechoslowakischen Industriebetrieb hin und wieder noch als Ort rigider Anweisungsunterworfenheit und totalitärer Kontrolle. ${ }^{45}$ Natürlich gab es auch „Diktatoren“ unter den Betriebsleitern, ${ }^{46}$ aber die Quellen berichten in der Masse von etwas ganz anderem: Der Direktor der Automobilwerke in Liberec beklagt sich bei einem Gewerkschaftsfunktionär, er habe in drei Monaten 80 Anweisungen erlassen, aber nicht eine einzige sei befolgt worden. ${ }^{47}$ Versuchten es die Betriebsleitungen mit „Durchgreifen“, handelten sie sich dafür in der Regel andere Probleme ein: Eine im Frühjahr 1953 „,von oben“ verordnete Normenerhöhung im Škoda-Zweigwerk in Smíchov, die zur Kürzung des Akkordsatzes führte, zog zum einen go-slow-Aktionen und einen Einbruch der Planerfüllung nach sich, zum anderen wachsende Produktion von Ausschuss, da ein Teil der Belegschaft das Arbeitstempo anzog, um so viel zu verdienen wie vor dem Lohnabzug. ${ }^{48}$ Wenn eine Verschärfung der Normen diskussionslos verfügt wurde, konnte es - noch lange nach der kommunistischen Machtübernahme - auch rabiat zugehen: Ohne viel Federlesens zu machen, vertrieben die Arbeiter in solchen Fällen Normierer und Kalkulatoren aus der Werkstatt. ${ }^{49}$

Derartige Erfahrungen werden es nahe gelegt haben, dass sich die Leitungskader qua Planerfüllungspakt zu arrangieren suchten. Einen Erfolg versprechenden Ansatzpunkt bot die Schichtarbeit, die - so der Protest der Arbeiter - ,ihr gesellschaftliches und Familienleben“ zerstörte. ${ }^{50}$ Im Juli 1962 beschloss das südmährische Kreiskomitee der KPTsch die stärkere Auslastung der zweiten und dritten Schicht und legte einen

40 Státní oblastní archiv (künftig zitiert: SOA), Prag. SONP, Karton 392, 1948-1954 (diverse Dokumente), Vermerk über solche Fälle, in denen Beschäftigte unserer Betriebe Streikdrohungen zur Durchsetzung von Lohnforderungen anwenden (Juli 1948).

41 Aus Dutzenden von Beispielen sei nur auf dieses hingewiesen: NA. Fonds 014/12, Bd. 31, Nr. 1249, Die Situation im Betrieb Slovenka Banská Bystrica (November 1958).

42 Kalinová, Ke změnám, 151.

43 Ebd., 153.

44 In der Region Moravská Ostrava wurde einem Arbeit suchenden Bergmann in einer Grube erklärt, man habe keine freie Stelle, nur die des Direktors. Der Bergmann antwortete, diese Stelle wolle er nicht, denn er suche eine Beschäftigung ,für längere Zeit“. VOA. ÚRO-PL, Karton 15, Inventarnummer 44/1, Stenographisches Protokoll der Plenarsitzung des Zentralrates der Gewerkschaften vom 19.-20. 10. 1956.

45 Madry, J. (2001): Entscheidungsfindung in der Tschechoslowakei nach Stalins Tod, in: Jan Foitzik (Hg.), Entstalinisierungskrise in Ostmitteleuropa 1953-1956. Vom 17. Juni bis zum ungarischen Volksaufstand. Politische, militärische, soziale und nationale Dimensionen. Paderborn 2001, 215.

46 VOA. ÚRO-Org., Karton 154, Inventarnummer 515, Bericht der Delegation des Kreiskomitees des Gewerkschaftsverbandes Maschinenbau in Liberec vom 11. bis 13. 10. 1956 (Betrieb Tatra Česká Lípa).

47 VOA. Celostátní akce, Karton 8, Inventarnummer 55, Beratung der Kreissekretäre [der Gewerkschaften] und der Abteilungsleiter des Zentralrates der Gewerkschaften am 29. 5. 1957.

48 VOA. ÚRO-VMP, Karton 2, 1953, Berichte der Brigaden und Berichte über sozialistische Wettbewerbe, Betrieb Škoda-Smíchov.

49 VOA. ÚRO-Sekr. II, Karton 66, Inventarnummer 215/1, Bericht über den Verlauf des Aktivs der Vorsitzenden der Lohnkommissionen bei den Betriebsausschüssen der ROH des Gewerkschaftsverbandes der Beschäftigten der Hütten und Erzgruben und der Lohnabteilungsleiter der Hüttenbetriebe im Kreis Ostrava am 14. 9. 1956.

50 Zit. nach Pick, M. Proč zyšovat směnnost ?, in: Odborář 18 (1965), Nr. 26, 1261-1266. 
Schichtbelegungskoeffizienten von $1,8^{51}$ fest (dieser sollte bis zum XII. Parteikongress der KPTsch im Dezember des gleichen Jahres erreicht werden). Das industrielle Management mehrerer südmährischer Maschinenbaufabriken hielt diesen Koeffizienten für ,irreal“ und reduzierte ihn deshalb zunächst für eine Fabrik in Kuřim auf 1,55. Dann überprüften die Werkstätten dieser Fabrik auf Veranlassung der Direktoren „,ihre Möglichkeiten“ und korrigierten den Koeffizienten noch einmal nach unten $(1,45) .{ }^{52}$

Einen Beitrag zur Kooperation von Arbeitern und Betriebsleitung im Planerfüllungspakt leistete offensichtlich die Solidarität der Arbeiter mit ihren zum Betriebsdirektor aufgestiegenen früheren Kollegen. Man könne doch nicht den „Arbeiterdirektor" anklagen, weil es an sozialen Einrichtungen im Betrieb fehle, meinte ein Bauarbeiter in Jindřichův Hradec. ${ }^{53}$ Den eigentlichen Ansatzpunkt des Paktes bildeten jedoch die Mängel der zentralen Planwirtschaft. Es sei gar nicht einzusehen, meinten Mitarbeiter der Lohnabteilung einer Brünner Maschinenbaufabrik, warum die Arbeiter wegen stockender Materialzufuhr zu dem niedrigeren Tarif für Wartezeiten arbeiten und für die Mängel des Plansystems draufzahlen sollten. ${ }^{54}$ Die materiellen Einbußen durch Wartezeiten wurden aus diesem Grunde - nicht nur im Maschinenbau - durch „Aufweichung“ der Arbeitsnormen ausgeglichen. ${ }^{55}$ Regierungsverordnungen, die diese Praxis unterbinden wollten, blieben mehr oder weniger auf dem Papier. ${ }^{56}$ Die Lohnbuchhaltungen dehnten solche Kompensationen auf ganze Arbeitskollektive aus: Zeichnete sich ab, dass eine Werkstatt oder Produktionsabteilung den Plan nicht erfüllen würde, wurden ihr Leistungen derjenigen Werkstätten überschrieben, die besser im Rennen lagen. ${ }^{57}$ Die innerbetrieblichen Beziehungen erschienen daher manchem Beobachter als „Familienwirtschaft": Alle seien „nett" zueinander. ${ }^{58}$

Zweifellos floss über die Kooperation zwischen den Lohnabteilungen der Betriebe und den Lohnkommissionen der Betriebsräte die egalitäre Orientierung der Betriebsräte in die betriebliche Lohnpolitik ein. Gegen die schon erwähnte „Aufweichung“ der Leistungsnormen schritt die Betriebsleitung offenbar nur in „schreienden Fällen“ ein. ${ }^{59}$ Ebenso spielte sich mit Zustimmung des Leitungspersonals ein, dass Überstunden, die in erheblichem Umfang bei der regelmäßigen „Planschlusspanik“ (šturmovština) anfielen,

51 Dieser Koeffizient gab die Verteilung der Belegschaft auf die drei Schichten an: Je höher der Koeffizient, umso stärker die Auslastung der Nachmittags- und der (höchst unattraktiven) Nachtschicht. Berechnet wurde der Koeffizient als Quotient aus der durchschnittlichen Gesamtzahl aller anwesenden Arbeiter und der durchschnittlichen Gesamtzahl aller in der Hauptschicht (6-14 Uhr) anwesenden Arbeiter.

52 VOA. Strojírenství, Karton 55, 1962, Bericht des Kreiskomitees des Gewerkschaftsverbandes für Maschinenbau des südmährischen Kreises über die Nutzung der Grundfonds durch Schichtarbeit.

53 VOA. KOR, Karton 75/1956, Inventarnummer 138/1, Bewertung der allgewerkschaftlichen Aktive der Bezirksfunktionäre vom 6. bis 20. 7. 1956 in allen Bezirken des Kreises České Budějovice.

54 VOA. ÚRO-PaM, Karton 8, 1957, Berichte der Lohnabteilung, Bericht über die Lohnverhältnisse im Maschinenbau.

55 Ebd. Für den Maschinenbau im Kreis Olomouc s. dazu: VOA. Strojírenství, Karton 35-36, Faszikel 1, 1957, Bericht über den Stand der Erfüllung der Aufgaben im Kreiskomitee des Gewerkschaftsverbandes Maschinenbau in Olomouc und die Situation im Betrieb Sigma Lutín vom 21. bis 31. 1. 1957.

56 VOA. ÚRO-Sekr., Karton 76, Inventarnummer 672, 1955, Bericht über die Maßnahmen zur Beseitigung von Mängeln bei der Entlohnung der Arbeit und zum Entwurf der politisch-organisatorischen Absicherung dieser Maßnahmen.

57 VOA. Strojírenství, Karton 26/1956, Unterlagen für die Sitzung des ZK des Gewerkschaftsverbandes der Beschäftigten des Maschinenbaus am 3. 2. 1956, Bericht der Delegation in den Betrieb Sigma, Nationalunternehmen Hranice, vom 25.-26. 1. 1956.

58 VOA. ÚRO-Sekr., Karton 66, Inventarnummer 215/1, Bericht über Vorbereitung und Verlauf der Diskussion über die Lohnpolitik im Betrieb Rudé právo in Větřní am 24. 4. 1956.

59 NA. Fonds 02/4, Bd. 19, Nr. 143, 23. 8. 1950, Bericht über ČKD Stalingrad (schwerer Maschinenbau); VOA. ÚRO-Sekr., Karton 75, Inventarnummer 240/5, Information für den Genossen Hnilička über den Stand der Verkürzung der Arbeitszeit vom 13. 11. 1956. 
denjenigen Arbeitern vorbehalten wurden, die mit „Hungerlöhnen“ auskommen mussten. ${ }^{60}$ Oft dienten die Kompensationen allein dem Zweck, die Ruhe aufrechtzuerhalten: In politisch bewegten Zeiten - etwa im Herbst 1956 - häuften sich von den Betriebsleitungen veranlasste Ausnahmen von den geltenden Lohnrichtlinien. ${ }^{61}$

Diejenigen, die mit der „,organisierten Unordnung ${ }^{\text {“62 }}$ aufräumen wollten, mussten sich meistens mit dem Stoßseufzer „Der Himmel ist hoch, und Prag ist weit! "63 begnügen. Erkundigten sich höhere Partei- und Gewerkschaftsfunktionäre nach der Situation im Betrieb, erhielten sie unbestimmte Antworten. Betriebsrat und Betriebsleitung deckten sich gegenseitig. ${ }^{64}$ Vermutete der Betriebsrat bei Abordnungen des Zentralrates der Gewerkschaften allzu bohrende Wissbegier, wurden sie erst gar nicht in den Betrieb hereingelassen. ${ }^{65}$ Die Gewerkschaftsführung neigte bei ihrer Kritik der „Unordnung“ gelegentlich zu einem nachsichtigen Blick zurück auf kapitalistische Verhältnisse, als es in den Betrieben, so argumentierte sie, wie am Schnürchen lief. ${ }^{66}$ Was dem Verdikt des gewerkschaftlichen Sozialismus-Konzepts verfiel, das in erster Linie Autorität und die Parole „Sozialismus heißt viel arbeiten“ favorisierte, kann allerdings auch unter einem Aspekt betrachtet werden, der andere politische Interessen in den Mittelpunkt stellt (s.u.).

3. Das den Betrieb durchdringende informelle Geflecht war gleichbedeutend mit organisatorischer (und funktionaler) Entdifferenzierung. Im vorliegenden Fall spricht wenig für die verbreitete These, Entdifferenzierung, d.h. die Auflösung differenzierter und institutionell verselbständigter Wertsphären und die damit einhergehende soziale Amorphie und „Atomisierung“ habe kommunistischen Machtansprüchen in die Hände gespielt, ja sei eine zentrale Voraussetzung der dauerhaften Festigung der Macht der kommunistischen Partei gewesen. ${ }^{67}$

Zunächst nutzte die Masse der Arbeiterschaft die schwindende Rigidität organisatorischer Integration auf Betriebsebene zur Herstellung von Machtverhältnissen, die sich langfristig immer weniger auf die offiziellen Strukturen stützten und immer mehr den authentischen politischen Willen der Belegschaften ausdrückten. Die Wahlen zu den Gewerkschaftsorganisationen in den Betrieben bieten dafür zahlreiche Beispiele: Den „Stars“ der Arbeiterklasse, also den gefeierten, mit Orden überhäuften Stoßarbeitern und Stachanovcen, die von einer internationalen Arbeiterkonferenz zur anderen eilten und sich

60 VOA. ÚRO-Sekr. II, Karton 66, Inventarnummer 215/1, Bericht über den Verlauf des Aktivs der Vorsitzenden der Lohnkommissionen bei den Betriebsausschüssen der ROH des Gewerkschaftsverbandes der Beschäftigten der Hütten und Erzgruben und der Lohnabteilungsleiter der Hüttenbetriebe im Kreis Ostrava am 14. 9. 1956.

61 VOA. ÚRO-VMP, Karton 6, 1956, Ergebnisse und Erfahrungen der Verkürzung der Arbeitszeit im Bereich des Industrieministeriums seit dem 1. 10. 1956 (für die 42. Sitzung des Präsidiums des ZK des Gewerkschaftsverbandes der Beschäftigten im Bergbau am 6. 12. 1956).

62 Das Zitat nach dem Bericht über die Tätigkeit des Betriebsrates im Betrieb Kovosvit in Třebíč vom 3. 9. 1952. VOA. ÚRO-Org., Karton 126, Inventarnummer 436.

63 VOA. ÚVOS-Horníci, Karton 7, 1949, Bericht über die Förderung, die sozialpolitischen Probleme, die Lohnverhältnisse, die Wohnungs- und Versorgungssituation und den Gesundheitszustand der Belegschaften im nordböhmischen Braunkohlenrevier vom 24. 8. 1948.

64 VOA. ÚRO-Org., Karton 157, Inventarnummer 523/1, Bericht [des Kreisgewerkschaftsrates Brünn] über den Verlauf der Wahlen [zu den betrieblichen Gewerkschaftsorganen] im Kreis Brünn vom 19. 10. 1956.

65 VOA. ÚRO-PaM, Karton 1, 1951, Untersuchungen zu den Schwierigkeiten bei der Eingliederung von Frauen in den Arbeitsprozess (Februar 1951).

66 VOA. ÚRO-Před., Karton 7, Inventarnummer 113, Stenographisches Protokoll der Sitzung des Präsidiums des Zentralrates der Gewerkschaften am 16. 11. 1950; Karton 16, Inventarnummer 177, Protokoll des Verlaufs der 7. Sitzung des Präsidiums des Zentralrates der Gewerkschaften am 28. 5. 1953.

67 So für die DDR Lepsius,M. R.: Die Institutionenordnung als Rahmenbedingung der Sozialgeschichte der DDR, in: Sozialgeschichte der DDR, 17-30. 
kaum noch im Betrieb blicken ließen ${ }^{68}$, wurde bei diesen Wahlen ebenso ein Denkzettel verpasst wie dem übereifrigen Normierer und dem allzu orthodoxen Mitglied der kommunistischen Partei. ${ }^{69} \mathrm{Zu}$ Mitgliedern von Deputationen konnten diejenigen frei gewählt werden, die das allgemeine Vertrauen ihrer Arbeitskollegen genossen, und an den Beratungen über die Beilegung von Streiks nahmen - wenn sich die Belegschaft energisch dafür einsetzte - auch Delegierte der Arbeiter teil, die ihr Mandat nicht der Kaderakte oder anderen politischen Konstellationen verdankten. ${ }^{70}$

Fehlende organisatorische Abgrenzung und mangelnde institutionelle Differenzierung auf Betriebsebene bedeuteten, dass Betriebsleitung, die betriebliche Partei- und die Gewerkschaftsorganisation alle das Gleiche machten bzw. mit dem „Gesamtproblem“ beschäftigt waren, d.h. mit der Planerfüllung. Der Betriebsdirektor besucht „sein“ Ministerium und kehrt mit 60 Waggonladungen Rohstoffen zurück, ${ }^{71}$ die Vorsitzenden der KPTsch-Organisationen in den Pilsener Škoda-Werken regeln in Prag mit den zentralen Behörden ebenfalls die Lieferung von Material (wobei sie sich allerdings als „Planbearbeiter“ tarnen), ${ }^{72}$ und der Betriebsratvorsitzende der Waggonbau-Fabrik Tatra-Butovice versteht sich so gut auf das Geschäft des Antichambrierens, dass die gesamte Belegschaft ihn hofiert und ein ums andere Mal zu den staatlichen Verwaltern der Ressourcen entsendet. ${ }^{73}$

Die Kritik an diesem Handlungsmuster ist bekannt: Da alle Handlungsorientierungen an ein gemeinsames Ziel rückgebunden waren, also nicht mit Entscheidungsalternativen einhergingen, können die Aktivitäten der betrieblichen Vertreter nicht als konkurrierende Handlungsstrategien bezeichnet werden. Erst diese wären imstande gewesen, soziale Dynamik freizusetzen. ${ }^{74}$

4. Ganz im Gegensatz zu solchen Annahmen war das informelle Beziehungs- und Einflussnetzwerk in den tschechoslowakischen Industriebetrieben der Ort einer dynamischen Umschichtung der Machtverhältnisse zugunsten der betrieblichen Organisationen der Gewerkschaften. Partei- und Gewerkschaftsführung standen diesem Vorgang, der bald nach dem Februar 1948 einsetzte, im Grunde ratlos gegenüber. Da beiden spätestens nach den landesweiten Unruhen und Streiks Anfang Juni 1953 klar war, dass „Dreinschlagen“ nichts nutzte, ${ }^{75}$ bereits vor 1953 jedoch auf Betriebsebene keine Instanzen zur wirksamen politischen und gewerkschaftlichen Formierung der Arbeiter

$68 \mathrm{Zu}$ diesem Typus, der „überall“ war, nur nicht am Arbeitsplatz, vgl. den Bericht über die Tätigkeit des Kreiskomitees Olomouc des Gewerkschaftsverbandes der Beschäftigten der Metallindustrie vom 1. 1. 1951 bis 30. 9. 1951. VOA. Strojírenství, Karton 5, Faszikel 1.

69 VOA. ÚRO-Org., Karton 109, Inventarnummer 384 e, Bericht über die Absicherung der Wahlen in die Gewerkschaftsorgane in den Lenin-Werken [Škoda-Werken].

70 VOA. ÚRO-Před., Karton 16, Inventarnummer 177, Protokoll über den Verlauf der 7. Sitzung des Präsidiums des Zentralrates der Gewerkschaften am 28. 5. 1953; Peter Heumos, Zum industriellen Konflikt in der Tschechoslowakei 1945-1968, in: Peter Hübner/Christoph Kleßmann/Klaus Tenfelde (Hg.), Arbeiter im Staatssozialismus. Ideologischer Anspruch und soziale Wirklichkeit. Köln-Weimar-Wien 2005, 473-497 (Zeithistorische Studien 31).

71 VOA. Strojírenství, Karton 10, Faszikel 2, 1954, Protokoll des Aktivs der Betriebsratvorsitzenden [der Maschinenbau-Fabriken] am 11. 2. 1954 in Prag.

72 NA. Fonds AN, Bericht über die Untersuchung der Arbeit der Partei in Pilsen im Zusammenhang mit den Ereignissen vom 1. 6. 1953 (nicht datiert).

73 VOA. Strojírenství, Karton 5, Faszikel 2, 1952, Bericht einer politischen Brigade des Zentralrates der Gewerkschaften über die Tätigkeit des Betriebsrates in der Waggonbau-Fabrik Tatra Studénka.

74 So lässt sich die Argumentation von Lepsius für die DDR zusammenfassen.. Vgl. dazu seinen in Anm. 67 zit. Aufsatz.

75 Wenige Wochen nach der Niederschlagung der Juni-Revolte durch Polizei, Volksmilizen und Einheiten der Grenzwache und der Armee flackerten unter den Bergleuten im nordböhmischen Braunkohlenrevier erneut Streiks auf. Vgl. Heumos, P. (2000): Dělnické stávky v Československu v padesátých letech, in: Pohledy 8 (2000), H. 6, 20-21. 
errichtet werden konnten ${ }^{76}$ und solche Bestrebungen nach 1953 noch weit mehr ins Leere stießen, ${ }^{77}$ blieben der Parteiführung und dem Zentralrat der Gewerkschaften im Wesentlichen Überzeugungsarbeit und, wenn man so will, die traditionellen Waffen der Bolschewiki: Geduld und Ironie. Die Zeit arbeitete daher für die Betriebsräte bzw. ihre Nachfolgeorganisationen, die Betriebsausschüsse der $\mathrm{ROH},{ }^{78}$ und als gegen Ende der sechziger Jahre im Zuge der gesellschaftlichen Reformbewegung („,Prager Frühling“) die Reorganisation der Einheitsgewerkschaft auf die Tagesordnung gesetzt wurde, hatte sich die Machtposition der betrieblichen Gewerkschaftsorganisationen längst so gefestigt, dass sie für partielle Verbesserungen nicht mehr zu haben waren und die Auflösung der Organisationsstrukturen der Einheitsgewerkschaft selbst in die Hand nahmen (s.u.).

Der erste Schritt zur Verschiebung der Machtverhältnisse im Betrieb war die Zurückdrängung der Betriebszellen der KPTsch, die anfangs das Kommando sowohl über die Betriebsleitung als auch über den Betriebsrat beanspruchten, da sie - so die Beschwerde mährischer Gewerkschaftsfunktionäre - das Prinzip der führenden Rolle der Partei in den Betrieben ,nicht richtig begriffen hatten.“ “79 Die Ambitionen der Parteizellen stießen beim Leitungspersonal wie bei den Betriebsräten auf energischen Widerstand. ${ }^{80}$ Die Partei sei ,an den Arbeitsplätzen fast nicht zu spüren," wurde schon 1951 für große Betriebe wie die Molotov-Eisenwerke in Třinec festgestellt. ${ }^{81}$

Auch bei der Wahrnehmung der Aufgabe, die den Betriebszellen der Partei qua Betriebsverfassung übertragen wurde, nämlich das industrielle Management $\mathrm{zu}$ kontrollieren, hatten die Betriebszellen einige Mühe, ihre Position zu behaupten. Der Informationsfluss im Planerfüllungspakt lief an ihnen vorbei, und sie mussten sich häufig eingestehen, dass sie keinen Einfluss auf die Betriebsorganisation der Gewerkschaften besaßen, schließlich sogar - wie in den Škoda-Werken - in Unkenntnis relevanter innerbetrieblicher Vorgänge mit ihren Stellungnahmen und Instruktionen zum Produktionsprozess die Partei „lächerlich machten“. ${ }^{82}$

Auf lange Sicht gewichtiger für die Herausbildung der informellen Machtkonstellation im Betrieb war die verbreitete Substituierung der Betriebsleitungen durch die Betriebsräte bzw. die Betriebsausschüsse der ROH. „Die Fragen der Produktion werden in den Sitzungen des Betriebsrates geregelt," beobachtete ein Gewerkschaftsfunktionär im Sigma-Pumpenwerk in Lutín (1950). ${ }^{83}$ In den Flugzeugwerken in Rýnovice klärte der Betriebsrat Produktionsprobleme ebenfalls in eigener Regie, „ohne die Werksleitung “. ${ }^{84}$ Bereits 1951 war es im Einzugsbereich des Kreisgewerkschaftsrates Prag eine allgemeine Erscheinung, dass die Betriebsräte „die Aufgaben der Betriebsleitung

76 Zur defizitären Heranbildung politischer Kader in den Betrieben: NA. Fonds 100/2, Bd. 4, Nr. 54, Bericht Káňa über die Situation in der Grube Präsident Gottwald im Revier Ostrava-Karviná (August 1951).

77 Heumos, P. (2004): Stalinismus in der Tschechoslowakei. Forschungslage und sozialgeschichtliche Anmerkungen am Beispiel der Industriearbeiterschaft, in: Journal of Modern European History , vol 2 (2004), No. 1, 82-109.

78 Diese entstanden 1959 durch die Zusammenlegung der Betriebsräte und der ebenfalls seit 1945 bestehenden Betriebsgruppen der Einheitsgewerkschaft. Text des Gesetzes vom 8. 7. 1959 in: Informační bulletin Ústřední rady odborů, Nr. 15/1959, $9 \mathrm{f}$.

79 VOA. KOR, Olomouc, Karton 14, 1950, Inventarnummer 59, Schreiben des Kreisgewerkschaftsrates Olomouc an den Sekretär des Zentralrates der Gewerkschaften Šmídmajer vom 12. 4. 1950.

80 SOA. SONP, Karton 10, 1949-1960, Protokoll der Konferenz der Abteilungen für kader- und sozialpolitische Fragen des gesamten Unternehmens [ d.h. der Vereinigten Stahlwerke Kladno] am 15. 3. 1949.

81 NA. Fonds 100/1, Bd. 15, Nr. 96, Untersuchung in den V.M. Molotov-Eisenwerken in Třinec (August 1951).

82 Škoda-Archiv. PV KSČ 2/238, Bericht über die Ausübung der Kontrolle der Betriebsleitung durch die betriebliche Parteiorganisation für das Unternehmenskomitee der KPTsch (Anlage zum Protokoll der Sitzung des Unternehmenskomitees der KPTsch am 23. 5. 1958).

83 VOA. KOR, Karton 14/1950, Inventarnummer 59, Bericht über die Untersuchung des sozialistischen Wettbewerbs.

84 Informační bulletin Ústřední rady odborů, Nr. 3/1952, 13. 
übernommen haben“ ${ }^{85}$ Die Gewerkschaftsführung konstatierte 1955 missbilligend in allen Sektoren der Industrie „häufige Fälle“, in denen „,die Werkstatträte die Wirtschaftsorgane ersetzen". ${ }^{86}$ Aus dem Kreisgewerkschaftsrat in České Budějovice war im folgenden Jahr noch einmal zu hören: „Es ist eine Tatsache, dass die Gewerkschaften in den Betrieben die Aufgaben der Betriebsökonomen übernehmen. Sie ersetzen die Betriebsökonomen [... $]^{\text {‘.87 }}$

$\mathrm{Zu}$ dieser Zeit hing die innerbetriebliche Reputation der Betriebsräte bereits entscheidend davon ab, in welchem Maße sie den Produktionsprozess in Gang halten konnten: „Wenn sich die Gewerkschaftsorganisation nicht darum kümmert, dass die Leute in den Betrieben Arbeit haben, " hieß es im Kreisgewerkschaftsrat Liberec, dann werde sie sich „,keine Autorität“ erwerben. ${ }^{88}$ An denjenigen, die für diese Autorität sorgten, fehlte es nicht. Ein Beispiel ist der Betriebsratvorsitzende der Prager Flugzeugwerke Rudý Letov, der mit Lieferfirmen verhandelte und durch das Land reiste, um Fragen zu klären, die „ausschließlich in die Kompetenz der Betriebsleitung fielen“. ${ }^{89}$ Hatten die Betriebsdirektoren die Mitglieder der Betriebsräte 1945 noch als „Idealisten“ verspottet, denen die „erforderlichen fachlichen Kenntnisse und Erfahrungen mit der Leitung eines Unternehmens fehlen" ${ }^{90}$ so musste ein Mitglied des Zentralrates der Gewerkschaften nach einer Inspektionsreise durch südmährische Industriebetriebe im Sommer 1956 einräumen, dass „die Gewerkschaftsfunktionäre in den Betrieben die wirtschaftlichen Probleme gut beherrschen. Manch einer redet wie ein gelernter Ökonom“".

Macht und Kompetenzen mussten den Betriebsleitungen offenbar nicht gewaltsam entrissen werden. Ihre Defizite und ihre schwache Position lagen so offen zutage (s.o.), dass sich die Gewerkschaften immer wieder veranlasst sahen, zur Geduld mit ihnen aufzurufen: Fachlich und politisch fähige Betriebsdirektoren und Ingenieure würden „nicht vom sozialistischen Himmel fallen".92 Fest in der Hand hatten die Vertretungsorgane der Gewerkschaften in den Betrieben die Arbeitsorganisation, und gerade diese Machtposition fiel ihnen kampflos zu. Das Einfallstor bildeten die unteren Chargen des Leitungspersonals, die formell für die Arbeitsorganisation zuständigen Werksstattleiter und Meister. ${ }^{33}$ Nach dem Zweiten Weltkrieg wurden diese wegen ihres Verhaltens in der Zwischenkriegsrepublik und - ob zu Recht oder nicht - auch aufgrund ihres Umgangs mit den Arbeitern unter der

85 VOA. ÚRO-Org., Karton 112, Inventarnummer 392/21, Bericht über die Arbeit der Instrukteure des Kreisgewerkschaftsrates [Prag] bei der Sicherstellung der Planerfüllung und bei den Vorbereitungen zum 4. Jahr des Fünfjahresplans vom 12. 12. 1951.

86 VOA. ÚRO-Před., Karton 21, Inventarnummer 212/2/1, Bewertung der Jahresmitgliederversammlungen, der Wahlen der Gruppen-Vertrauensmänner, der Werkstatt- und Betriebsräte (für die Sitzung des Präsidiums des Zentralrates der Gewerkschaften am 13. 1. 1955).

87 VOA. KOR, Karton 75/1956, Inventarnummer 138/3, Protokoll der Plenarsitzung des Kreisgewerkschaftsrates České Budějovice am 18. 5. 1956.

88 VOA. KOR/1956, Karton 80, Inventarnummer 143, Protokoll der Sitzung des Präsidiums des Kreisgewerkschaftsrates Liberec am 4. 5. 1956.

89 VOA. ÚRO-Org., Karton 138, Inventarnummer 467, Berichte der Brigaden des Zentralrates der Gewerkschaften über die Kontrolle des Verlaufs des sozialistischen Wettbewerbs (Materialien für die Plenarsitzung des Kreisgewerkschaftsrates Prag am 21. 3. 1953).

90 SOA. ČKD-Ú, Karton 2, 30-44, Schreiben des Prokuristen der Generaldirektion der Böhmisch-mährischen Maschinenbauwerke in Prag an das Industrieministerium vom 4. 6. 1945.

91 VOA. ÚRO-Org., Karton 156, Inventarnummer 519/1-7, Bericht der Delegation Pokorný im Kreis Jihlava vom 3. bis 7. 7. 1956.

92 VOA. ÚRO-Org., Karton 138, Inventarnummer 470, Protokoll der Sitzung des erweiterten Präsidiums des ZK des Gewerkschaftsverbandes der Beschäftigten des schweren Maschinenbaus am 30. 1. 1953.

93 Im Folgenden stütze ich mich hauptsächlich auf diese Quellen: Škoda-Archiv. ZVIL 444, Př 336 [1958], Dokument über die Arbeit der Meister in den Lenin-Werken [Škoda-Werken] Pilsen; SOA. SONP, Karton 389, 1946-1962, Analyse der Arbeit der Meister [in den Vereinigten Stahlwerken Kladno] vom 12. 12. 1963; SOA. SONP, Karton 396, 1954-1966, Analyse der Entwicklung der Stellung der Meister im Nationalunternehmen Vereinigte Stahlwerke Kladno vom 19. 8. 1966. 
nationalsozialistischen Kriegswirtschaft im Protektorat Böhmen und Mähren als „kapitalistische Antreiber“ und als „asozial“ angeprangert und vielfach durch rasch angelernte Arbeiter und Vorarbeiter oder durch „Schichttechniker“, „Dispatcher" und „Schwarze“ (als Arbeiter entlohnte Meister) ersetzt - nicht ohne Zutun des industriellen Managements, ${ }^{94}$ das selbst mit einigen Schrammen aus der nationalsozialistischen Okkupation hervorgegangen war. ${ }^{95}$ Betriebsleitungen wie Arbeiter profitierten von dem angeschlagenen sozialen Prestige des Meisters. Notierte dieser die tatsächlichen Wartezeiten im Produktionsprozess, wurde er zum Direktor zitiert, der befürchtete, dass man ihm höheren Orts dieses Stück Realität ankreiden würde. ${ }^{96}$ Auf der anderen Seite wichen die Meister unter dem Druck der Arbeiter zurück und widmeten sich in vielen Betrieben ausgiebig der Beanstandung harter Leistungsnormen. ${ }^{97}$ Bei der Zuteilung der Arbeit wurden Anordnungen der Werkstattleiter und Meister häufig „,nicht respektiert“, und die Schmiede in den Pilsener Škoda-Werken, die aufgrund ihrer hohen Qualifikation traditionell eine starke Stellung im Betrieb besaßen, suchten sich die Arbeit aus, „die ihnen passt". ${ }^{98}$ „Onkelhafte“ Kontrolle durch die Meister ${ }^{99}$ zog innerbetriebliche „Wanderungsprozesse“ " nach sich, die sich von Arbeitsplätzen mit hoher Arbeitsintensität und vorwiegend technisch begründeten Normen zu Arbeitsplätzen mit niedrigerer Arbeitsintensität, einem hohen Anteil von Handarbeit und „,weichen“" Normen bewegten. ${ }^{100}$ Unter der Regie der „,neuen“ Werkstattleiter, deren soziale Distanz zu den Arbeitern gering war, entfalteten sich in kleineren Arbeitertrupps (čety) gut sortierte ,graue“ Märkte und eine reiche Subkultur des Aushandelns mit den Normierern und Angestellten der Lohnabteilungen der Betriebe, besonders bei der Festlegung des Zeitaufwandes für größere Aufträge. Die Mitglieder dieser Trupps pflegten streng darauf zu achten, dass „alle gleich bezahlt wurden“" und pochten bei Konfrontationen mit den betrieblichen Autoritäten auf „Gleichmacherei“ als positives Prinzip ihres sozialen Handelns. ${ }^{101}$

5. Der Prozess des Machterwerbs der Gewerkschaftsorganisationen, der sich außerhalb aller formalen Regelungen vollzog, die Partei- und Gewerkschaftsführung für die Organisation der Betriebe vorsahen und daher von ihrem ständigen Lamentieren über

94 SOA. SONP, Karton 10, 1949-1960, Protokoll der Konferenz der Abteilung für kader- und sozialpolitische Fragen des gesamten Unternehmens [Vereinigte Stahlwerke Kladno].

95 VOA. NHK, Karton 4, Inventarnummer 8, Betriebsrat der Textilfabrik Hybler (bei Semily) an den Zentralrat der Gewerkschaften vom 27. 7. 1945.

96 VOA. Celostátní akce, Karton 8, Inventarnummer 52, Stenographisches Protokoll der Beratung der ZK-Vorsitzenden der Gewerkschaftsverbände mit führenden Mitarbeitern des Zentralrates der Gewerkschaften in Prag am 3. 5. 1957

97 VOA. ÚRO-Před., Karton 64, Inventarnummer 381 III/1, Bericht über Lohnentwicklung und Arbeitsproduktivität im Jahr 1962, über die Verwirklichung der Beschlüsse des Präsidiums des Zentralrates der Gewerkschaften und über die Aufgaben der ROH bei der Entlohnung der Arbeit (Anlage II).

98 Škoda-Archiv. ROH 16/PV 164, Protokoll der Sitzung des Betriebsausschusses der ROH in der Schmiede am 10. 4. 1957.

99 Zit. nach dem Bericht über die Untersuchung in der Fabrik Epiag in Loket vom 27. 11. 1951. VOA. ÚRO-Org., Karton 111, Inventarnummer 387

100 Škoda-Archiv. ZVIL 1865/B 248, Bericht [des Unternehmensdirektors] für das städtische Komitee der KPTsch in Pilsen über die Probleme der Durchsetzung der Schichtarbeit, des ununterbrochenen Betriebes und der Überführung der Beschäftigten von Hand- zu Maschinenarbeit im Jahr 1962. - Für die Pilsener Werke ist der innerbetriebliche „Wanderungsprozess“ von der Maschinen- zur Handarbeit für den Zeitraum 1960-1968 auch statistisch belegt. Der Anteil der Maschinenarbeiter an der gesamten Arbeiterschaft der Werke fiel in diesem Zeitraum von 31 auf 25, 2 Prozent, der Anteil der Handarbeiter stieg von 69 auf 74, 8 Prozent. Vgl. dazu den Textund den Tabellenteil des Unterlagenmaterials für die programmatische Erklärung der Leitung des Nationalunternehmens Škoda zum Zeitraum 1960-1968 (Juli 1969). Škoda-Archiv. RP, Karton 10, Nr. 180.

101 Škoda-Archiv. ZVIL 179, osob 993, Protokoll der Verhandlung der Disziplinarkommission der Škoda-Werke Pilsen am 7. 6. 1951 (Aussagen Čechura, Pazák und Jelínek). 
die Verletzung des „,demokratischen Zentralismus“ begleitet wurde, ${ }^{102}$ fügt sich also nicht in das Erklärungsmuster, wonach institutionelle Entdifferenzierung die Verhaltensoptionen der „entsubjektivierten“ Bürger im Wesentlichen auf privates Räsonnement, Ritualisierung der geforderten Konformitätsgesten und systemwidrige „Nischen“ oder geduldete Netzwerke einschränkte. ${ }^{103}$ Die Entwicklung der Betriebsräte und - nach ihnen der Betriebsausschüsse der ROH ist ein Beispiel dafür, dass das soziale Handlungsfeld des Betriebes ein erhebliches Potential an Fähigkeiten zur Selbstorganisation bergen konnte und informelle Prozesse der Machtappropriation ermöglichte, die am Ende klar formulierte, auf breiter politischer Willensbildung beruhende gesellschaftliche Handlungsalternativen hervorbrachten.

Das war - wie oben schon angedeutet - im Jahr 1968 der Fall, als die Masse der Betriebsausschüsse der ROH verlangte, dass ihre seit vielen Jahren übliche informelle Praxis, nämlich den Betrieb zur „Festung“ gegen die zentralen Machtinstanzen auszubauen und die eigene Machtstellung im Betrieb zu konsolidieren, nun formell anerkannt und in Statuten und Gesetzen verankert wurde. Gefordert wurde der vollständige Rückzug der KPTsch aus dem Produktionsprozess und die Aufhebung des Machtmonopols, das die Partei auch über die Gewerkschaften ausübte; das Verbot von Interventionen der Gewerkschaftsführung in die Gewerkschaftsorganisationen in den Betrieben, die den Zentralrat nur noch als ihr Exekutivorgan zu tolerieren bereit waren; die Beschneidung der Kompetenzen der Betriebsleitung durch das Verbot von Maßnahmen, die sich gegen die „Interessen“ der Arbeiter richteten; die Bindung der Entscheidung über die Ausrufung eines Streiks an den Einzelbetrieb u.a.m. In eigener Regie verwirklichten die Betriebsausschüsse die Forderung nach Autonomie der Einzelgewerkschaften: Aus den insgesamt 13 Industriegewerkschaften der Einheitsgewerkschaft entstanden 25 Berufsgewerkschaften, die ihre Autonomie erklärten, verstanden in erster Linie als Veto gegen das „Hineinregieren“ von Partei- und Gewerkschaftsführung in ihre Belange. ${ }^{104}$

All dies war auch in der „reformierten“ und „liberalisierten“ KPTsch des Jahres 1968 nicht unterzubringen. Der daher zu erwartende grundsätzliche Konflikt kam allerdings nicht zur Austragung, da die Okkupation der Tschechoslowakei am 21. August 1968 andere Frontbildungen in den Vordergrund rückte. Dass dieser Konflikt prinzipiellen Charakter angenommen hätte, ist umso weniger zu bezweifeln, als die starke Stellung der Betriebsausschüsse nicht auf okkasionellem Machterwerb beruhte, sondern auf der Programmatik einer breiten syndikalistischen Strömung in der Arbeiterschaft seit 1945. Deren leitende Zielsetzung, nämlich die Inbesitznahme der Betriebe („Die Fabrik gehört uns allen“"105) durch die Aufhebung der Trennung von „Leitung und Ausführung“, wurde schon vor 1948 und danach u. a. im Betriebsrat der Škoda-Werke formuliert. ${ }^{106}$

102 VOA. ÚRO-Před., Karton 21, Inventarnummer 212/2/1, Bewertung der Jahresmitgliederversammlungen, der Wahlen der Gruppen-Vertrauensmänner, der Werkstatt- und Betriebsräte (für die Sitzung des Präsidiums des Zentralrates der Gewerkschaften am 13. 1. 1955).

103 Vgl. dazu den in Anm. 67 zit. Aufsatz von Lepsius.

104 Eingehend dazu Heumos, P.: Betriebsräte, Betriebsausschüsse der Einheitsgewerkschaft und Werktätigenräte. Zur Frage der Partizipation in der tschechoslowakischen Industrie vor und im Jahr 1968, in: Gehrke, B. - Horn, G.-R. (Hrsg.), 1968 und die Arbeiter. Studien zum ,proletarischen Mai“ in Europa. Hamburg 2007, 131-159.

105 Škoda-Archiv. ROH 13/669, Protokoll der Plenarsitzung des Betriebsrates vom 28. 5. 1951.

106 VOA. KOR Moravská Ostrava, Karton 1/1947, Inventarnummer 13, Protokoll der Arbeitssitzung des Präsidiums des Kreisgewerkschaftsrates, der Vorsitzenden und Sekretäre der Kreiskomitees der Gewerkschaftsverbände und der Sekretäre der Bezirksgewerkschaftsräte am 26. 8. 1947 in Ostrava; Škoda-Archiv. ZVIL 1515/PV 1287, Protokoll der gemeinsamen Sitzung des Betriebsrates und der Betriebsgruppe der ROH am 10. 1. 1950. 
In der Tat erschien die intendierte Auflösung des Gegensatzes von „Leitung und Ausführung" immer im Kontext anderer wesentlicher Merkmale des syndikalistischen Konzepts. Dazu gehörten vor allem: ein starker antistaatlicher, antizentralistischer und antibürokratischer Affekt mit dem Akzent auf „Degenerierung“ und „Unproduktivität“ der institutionellen Arrangements höherer Ordnung bei gleichzeitigem Eintreten für die Autonomie der einzelnen Arbeitergruppen; Vorbehalte gegen die politische Organisationsform der Partei, in gewissem Maße auch gegen die „Intellektuellen“ als eine vom Staat qua Erwerbschancen weitgehend abhängige soziale Gruppe; die hohe Wertschätzung der erzieherischen Arbeit der gewerkschaftlichen Basisorganisationen; die Betonung der Gleichwertigkeit aller „produktiven“ Arbeit einschließlich landwirtschaftlicher Tätigkeit etc.

Es geht hier nicht um den Syndikalismus als solchen. Vor dem Hintergrund einer bewussten gesellschaftlich-politischen Initiative lassen sich jedoch zum einen die aus der Vogelflug-Perspektive aufgenommenen Wirklichkeitsbilder der „Unordnung“ und „Fehlentwicklung“ in den Betrieben als tastende Schritte in einem Lernprozess bzw. als in mancher Hinsicht widersprüchliche - Anläufe zur Aneignung einer neuen sozialen Realität durch die Arbeiter interpretieren. Zum anderen steht die syndikalistische Entwicklung dafür, dass eine politische Aktion, der anfangs nicht mehr zur Verfügung stand als vom kommunistischen System im eigenen Interesse tolerierte Netzwerke und informelle Einfluss- und Austauschbeziehungen, enormes Gewicht erlangen konnte.

6. Die kompensatorischen Leistungen, die über das informelle betriebliche Aktionsfeld im Sinne einer beständigen Systemreparatur erbracht wurden, legten es Partei und Staat nahe, die Toleranzgrenzen gegenüber betrieblicher „Unordnung“ immer weiter hinauszuschieben. Zugleich leisteten Partei- und Gewerkschaftsführung selbst einen aktiven Beitrag zur Entdifferenzierung von Wertbeziehungen, organisatorischen Strukturen und spezifischen Rationalitätskriterien im Betrieb, indem sie Arbeits- und Lebenswelt entgrenzten. Es hapert mit sozialistischen Arbeitsinitiativen in den Vereinigten Stahlwerken Kladno, also werden die Kinder der Stahlwerker in der Schule aufgefordert, ihre Väter $\mathrm{zu}$ fragen, warum sie noch nicht als „,bester Arbeiter“ aus einem sozialistischen Wettbewerb hervorgegangen seien. ${ }^{107}$ Je länger je mehr musste sich dies - um hier als advocatus diaboli zu argumentieren - unter dem Gesichtspunkt der Stabilisierung von Herrschaft als kontraproduktiv erweisen: In Gesellschaften, die - wie der Staatssozialismus - durch den Primat hierarchischer Differenzierung gekennzeichnet sind, muss die Spitze der Hierarchie die Grenzen des Systems (in unserem Falle: des Systems „Betrieb“) kontrollieren können, um die Herrschaft nicht zu verlieren. Wenn jedoch die Außenbeziehungen des Systems komplexer werden, verringern sich die Kontrollmöglichkeiten.

107 VOA. ÚRO-Org., Karton 105, Inventarnummer 382, Bericht des Instrukteurs des Kreisgewerkschaftsrates Prag für Januar 1951. 


\title{
K otázce neformálních mocenských poměrů ve státním socialismu. Př́íklad československých průmyslových podniků v letech 1945-1968
}

\section{Peter Heumos}

\begin{abstract}
Abstrakt
Neformální mocenské vztahy ve státním socialismu sovětské provenience se daly najít především v průmyslu. Jejich rozsah byl v každé zemi odlišný a měl různé dopady na organizačně- politickou integraci průmyslových podniků do celého systému. Díky silnému postavení závodních rad před komunistickým převzetím moci a $\mathrm{v}$ důsledku slabé pozice vedení průmyslových podniků 50. letech vznikl v Československu po roce 1948 v průmyslových podnicích mírně syndikalistický proces samosprávy a mocenské apropriace průmyslových dělníků, který kulminoval během hospodářské reformy v 60 . letech. Zásadním požadavkem stažení KSČ a ÚRO z prrímého řízení výrobního procesu zahájil v roce 1968 zásadní systémový konflikt, který se ještě vyostřil tím, že reformní hnutí v průmyslových podnicích výrazně preferovalo zájmy a mocenskou pozici managementu. Okupace Československa v srpnu 1968 zabránila vyřešení tohoto systémového konfliktu.
\end{abstract}

Klíčová slova: komunismus; průmysloví dělníci; neformální mocenské struktury; závodní rady; podniková autonomie.

\section{Zur Frage informeller Machtverhältnisse im Staatssozialismus. Das Beispiel der tschechoslowakischen Industriebetriebe 1945-1968}

\begin{abstract}
Abstrakt
Informelle Machtverhältnisse im Staatssozialismus sowjetischer Provenienz waren vor allem im industriellen Bereich zu finden. Ihr Ausmaß differierte von Land zu Land und hatte unterschiedliche Folgen für die organisatorisch-politische Integration der Industriebetriebe in das Gesamtsystem. Gestützt auf die starke Stellung der Betriebsräte v o r der kommunistischen Machtübernahme und dank der schwachen Position des industriellen Managements in den 50er Jahren entwickelte sich in der Tschechoslowakei nach 1948 in den Industriebetrieben ein syndikalistisch eingefärbter, von den gewerkschaftlichen Betriebsorganisationen getragener Prozess der Selbstorganisation und Machtappropriation der Industriearbeiter, der im Zuge der Wirtschaftsreform der 60er Jahre kulminierte. Mit den zentralen Forderungen nach dem Rückzug der KPTsch und des ÚRO aus der direkten Steuerung des Produktionsprozesses bahnte sich 1968 ein grundlegender Systemkonflikt an, der dadurch angeheizt wurde, dass die Reformbewegung in den Industriebetrieben deutlich die Interessen und die Machtposition des Managements präferierte. Die Okkupation der Tschechoslowakei im August 1968 hat die Austragung dieses Systemkonflikts verhindert.
\end{abstract}

Key words: communism; industrial workers; informal power structures; shop steward; autonomy of industrial plants. 